\title{
Studies on the Compounds Related to PCB (V)
}

\author{
Detection and Determination of Unknown Organochlorinated \\ Compounds in Kanemi Rice Oil Caused the "Yusho"
}

\author{
(Received November 2, 1977) \\ Hideaki Miyata, Takashi Kashimoto and Nobuharu Kunita \\ (Osaka Prefectural Institute of Public Health 3-69, \\ Nakamichi 1-chome, Higashinari-ku, Osaka)
}

\begin{abstract}
It was considered that all the chlorine in Kanemi rice oils which caused the "Yusho" was derived from PCB, and the PCB concentrations were calculated from the concentration of total chlorine in the oils in 1969 to 1970.

However, in this study, the PCB concentrations in the oils were barely as $1 / 3$ to $1 / 7$ times as those which were calculated from the concentration of total chlorine. The difference between these two PCB levels suggests that unknown organochlorinated compounds will exist in large quantities in the oils. The oils were saponified, extracted with $n$-hexane, and the extracts were cleaned up by gel permeation chromatography and thin layer chromatography. Then, unknown organochlorinated compounds were analyzed by ECD-GC, GCMS and neutron activation analysis. On $2 \%$ OV-210 column, the gas chromatograms of these compounds showed many peakes with later R.T. than that of PCT (Aroclor 5460). GC-MS analysis revealed that the compounds were composed of penta- to deca-chlorinated quaterphenyl, hepta- to nona-chlorinated quaterphenyl ether and penta- to octa-chlorinated terphenyl. Polychlorinated quaterphenyls occupied quantitatively the most of the compounds. The result of neutron activation analysis showed a surprising fact, i.e., in Kanemi rice oils, which were manufactured on Feb. 5, 9 and 10 in 1968, these unknown organochlorinated compounds were contained 1.1, 3.6 and 4.1 times, respectively, more than PCB amounts. Therefore, it became clear that the causal compounds of the "Yusho" were not only PCB and PCDF but also the higher molecular chlorinated compounds.
\end{abstract}

\section{Introduction}

In 1968, the "Yusho," symptoms characterized by acne, pigment of the skin, and nervous and gastrointestinal disorder, affected over 13,000 people in Western Japan. Much efforts have been done to detect the causal compounds which gave rise the "Yusho." In 1969 to 1970, it became clear that the cause was the contamination by PCB in Kanemi rice oil used for cooking. ${ }^{1,2)}$ Moreover, polychlorodibenzofuran (PCDF) was detected in the oils in 1975. ${ }^{3}$ Although the ratios of PCDF vs. PCB concentration in the oils ranged $1 / 70$ to $1 / 209,{ }^{3,4}$ ) it was considered one of the causal factors, in view of its strong toxicity.

The concentration of total chlorine in Kanemi rice oil was determined by various methods, ${ }^{1,2}$ and reported that the concentration in the oil which was manufactured on Feb. 5th in 1968, when PCB first contaminated the oil, ranged about 1,000 to 1,500 ppm. ${ }^{1,2)}$ In those days, it was considered that all chlorine in the oil was derived from PCB, consequently the PCB concentrations of the oils were calculated from those of total chlorine, i.e., as PCB (Kanechlor 400) contained about 50\% chlorine, the PCB levels in the oils was estimated as 2,000 to 3,000 ppm. ${ }^{1,2)}$

On the other hand, we obtained the data of PCB $968 \mathrm{ppm}$ and $7.8 \mathrm{ppm}$ from Kanemi rice oil which was manufactured on Feb. 5 in 1968. The concentration was barely as $1 / 3$ times as the PCB concentration which was calculated from the concentration of total chlorine. The diffence between these two PCB levels suggests that unknown organochlorinated compounds will 
Table 1. The Concentration of Total Chlorine and PCB in Kanemi Rice Oils Caused the "Yusho"

\begin{tabular}{|c|c|c|c|c|c|}
\hline \multirow{2}{*}{ Production date } & \multicolumn{4}{|c|}{ Concentration of total chlorine (ppm) } & \multirow{2}{*}{$\begin{array}{l}\text { Concentration } \\
\text { of } \mathrm{PCB}^{\mathrm{a}} \text { (ppm) }\end{array}$} \\
\hline & Treatment & $\begin{array}{l}\text { Activation } \\
\text { analysis }\end{array}$ & $\begin{array}{l}\text { Fluorescene } \\
\text { X-ray analysis }\end{array}$ & $\begin{array}{c}\text { Chemical } \\
\text { analysis }\end{array}$ & \\
\hline Feb. 5th, 1968 & $\begin{array}{l}\mathrm{A} \\
\mathrm{B}\end{array}$ & $\begin{array}{c}1,485 \\
1,100 \sim 1,260^{1)} \\
997\end{array}$ & $1,100 \sim 1,300^{2)}$ & $1,020 \sim 1,500^{1)}$ & 500 \\
\hline Feb. 9th, 1968 & $\begin{array}{l}\text { A } \\
\text { B }\end{array}$ & $\begin{array}{l}554 \\
448\end{array}$ & $390^{2)}$ & & 80 \\
\hline Feb. 10th, 1968 & $\begin{array}{l}\text { A } \\
\text { B }\end{array}$ & $\begin{array}{l}446 \\
462\end{array}$ & $360 \sim 700^{2)}$ & & 83 \\
\hline
\end{tabular}

A, original oil; B, the hexane extract from the saponified oil

a) Shown as the chlorine concentration which were calculated from the concentration of PCB with the method of the authors ${ }^{\text {) }}$

Table 2. Compounds of Gas Chromatograph Peaks in Fig. 3

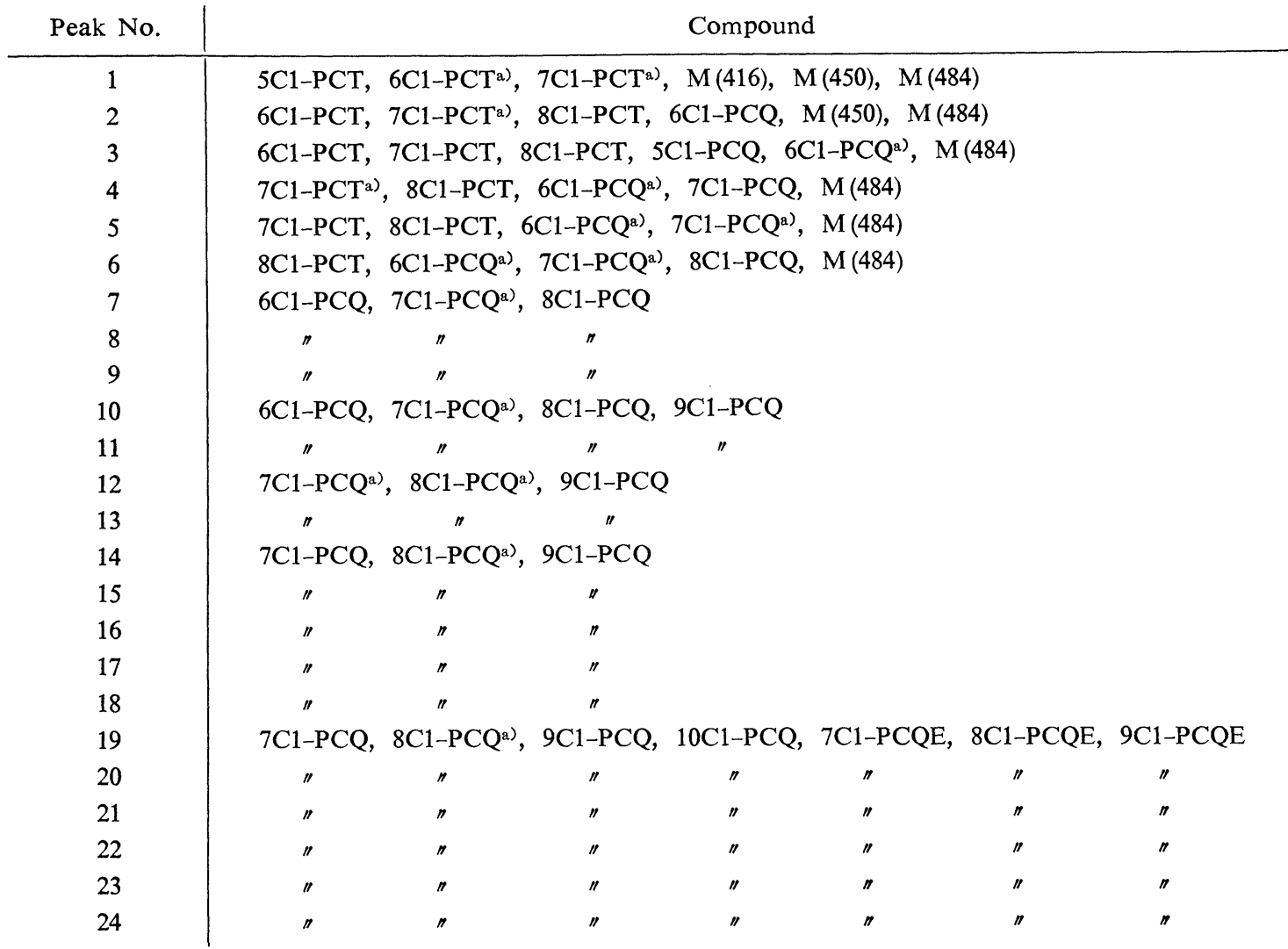

PCQ, polychlorinated quaterphenyl; PCQE, polychlorinated quaterphenyl ether

a) Main component 
be present in a large quantity in the oil. Moreover, the PCB intake by Yusho patients was based on the PCB concentration which was calculated from that of total chlorine in the oils. ${ }^{5}$ ) Therefore, thinking that the solution of above question is basically the most important thing to clarify the causal factors of "Yusho," we attempted to detect unkown organochlorinated compounds in Kanemi rice oil.

\section{Materials and Method}

\subsection{Materials}

Kanemi rice oils: The oils manufactured on Jan. 21st, Feb. 5, 9 and 10th in 1968 (K-1, K-2, $\mathrm{K}-3$ and $\mathrm{K}-4$, respectively), and their lot number were 12190546991, 821119079, 15520 and 16793, respectively, were employed.

\subsection{Reagents}

$2,3,4,2^{\prime}, 3^{\prime}, 4^{\prime}$-hexachlorobiphenyl $(99 \%$ in purity) and PCT (Aroclor 5460) were obtained from Analabs Inc. PCB (Kanechlor 400) preparation was distributed to our laboratory in 1972 by the Ministry of Health and Welfare. PCDF (containing $46.3 \sim 56.8 \%$ chlorine) and polychlorinated naphthalene (containing 50 59\% chlorine) were obtained from Wako Pure Chemical Co., Ltd. TLC plates were $20 \times 20 \mathrm{~cm}$ glass plates coated with Kieselgel $60 \mathrm{~F}_{254}$ of Merck Co., Ltd. Acetone, methylene dichloride, ethanol, ethyl ether, $n$-hexane, pottassium hydroxide and anhydrous sodium sulfate were of reagent grade for PCB analysis obtained from Wako Pure Chemical Co., Ltd.

\subsection{Analytical method}

Five grams of samples were saponified in 100 $\mathrm{ml}$ of $1.5 \mathrm{~N}$ alcoholic pottassium hydroxide solution by refluxing for $1 \mathrm{hr}$. Fifty $\mathrm{ml}$ of water was added to the saponified sample, and the aqueous solution was extracted 3 times with $100 \mathrm{ml}$ of $n$-hexane. The combined extract was washed 3 times with $100 \mathrm{ml}$ water, dried over anhydrous sodium sulfate, concentrated to $5 \mathrm{ml}$ and dried under a jet of nitrogen at $40^{\circ} \mathrm{C}$. The residue was dissolved with 7.5 to $15 \mathrm{ml}$ of methylene chloride. The $2.4 \mathrm{ml}$ of the methylene chloride solution was cleaned up by gel permeation chromatography (GPC). GPC condition was as follows: Column, two $50 \mathrm{~cm} \times 8 \mathrm{~mm}$ i.d. stainless column packed with Shimadzu HSG-15; eluent, methylene chloride; flow rate, $0.6 \mathrm{ml}$ / min; detector, UV $254 \mathrm{~nm}$; injection volume,
$0.2 \mathrm{ml}$. The GPC chromatogram of the oil showed five peaks.

The eluates corresponding to the fourth peak were collected, concentrated and dried under a jet of nitrogen. The residue was dissolved with a little quantity of acetone, and cleaned up by thin layer chromatography. Sample was spotted on TLC plate and developed with $n$-hexane, and the portion of UV band was scraped off with a spatula. It was then extracted by shaking with acetone, ethyl ether and $n$-hexane, respectively, and the combined extract was separated from adsorbent by filtering. The extract was concentrated, and unknown organochlorinated compounds were identified by GC and GC-MS.

The chlorine level of them was determined by neutron activation analysis. GC analysis was carried out on a Varian Aerograph 2100 equipped with ${ }^{63} \mathrm{Ni}-\mathrm{ECD}$. GC conditions: $1.8 \mathrm{~m}$ $\times 2 \mathrm{~mm}$ glass column packed with $2 \%$ OV-210 on Gaschrom Q (100/120 mesh); injection, detector and column temp., 215,293 and $245^{\circ} \mathrm{C}$, respectively: carrier gas, $\mathrm{N}_{2}(30 \mathrm{ml} / \mathrm{min})$. GCMS analysis was carried out on a Shimadzu LKB-9000 GC-MS. GC-MS conditions: $1 \mathrm{~m} \times$ $3 \mathrm{~mm}$ glass column packed with $2 \%$ OV-210 on Gaschrom Q (100/120 mesh); separator, inlet and column temp., 270,250 and $220^{\circ} \mathrm{C}$, respectively; ionizing voltage, $20 \mathrm{eV}$; accel voltage, $3.5 \mathrm{kV}$; carrier gas, $\mathrm{He}(30 \mathrm{ml} / \mathrm{min})$. Activation analysis: The samples were irradiated for 5 to 10 minutes in Triga Mark II Reactor at Rikkyo University with two neutron fluxes of $5 \times 10^{12} \mathrm{n} / \mathrm{cm}^{2} / \mathrm{sec}$ and $1.5 \times 10^{12} \mathrm{n} / \mathrm{cm}^{2} / \mathrm{sec}$ and the activities of ${ }^{38} \mathrm{Cl}$ were measured with a Canbera $\mathrm{Ge}(\mathrm{Li})$ detector. $2,3,4,2^{\prime}, 3^{\prime}, 4^{\prime}$-hexachlorobiphenyl was used as the standard of chlorine.

\section{Results}

As shown in Table 1, neutron activation analysis revealed that the concentration of total chlorine in Kanemi rice oils, which were manufactured on Feb. 5, 9 and 10th in 1968, were 1485, 554 and $446 \mathrm{ppm}$, respectively. These concentrations were of similar levels to those which had been determined by various methods. However, PCB concentration of each oil, which was showed as the chlorine concentration, was 500, 80 and $83 \mathrm{ppm}$, respectively, and these PCB concentrations were about $1 / 3,1 / 7$ and $1 / 5$ times level in comparison with the 


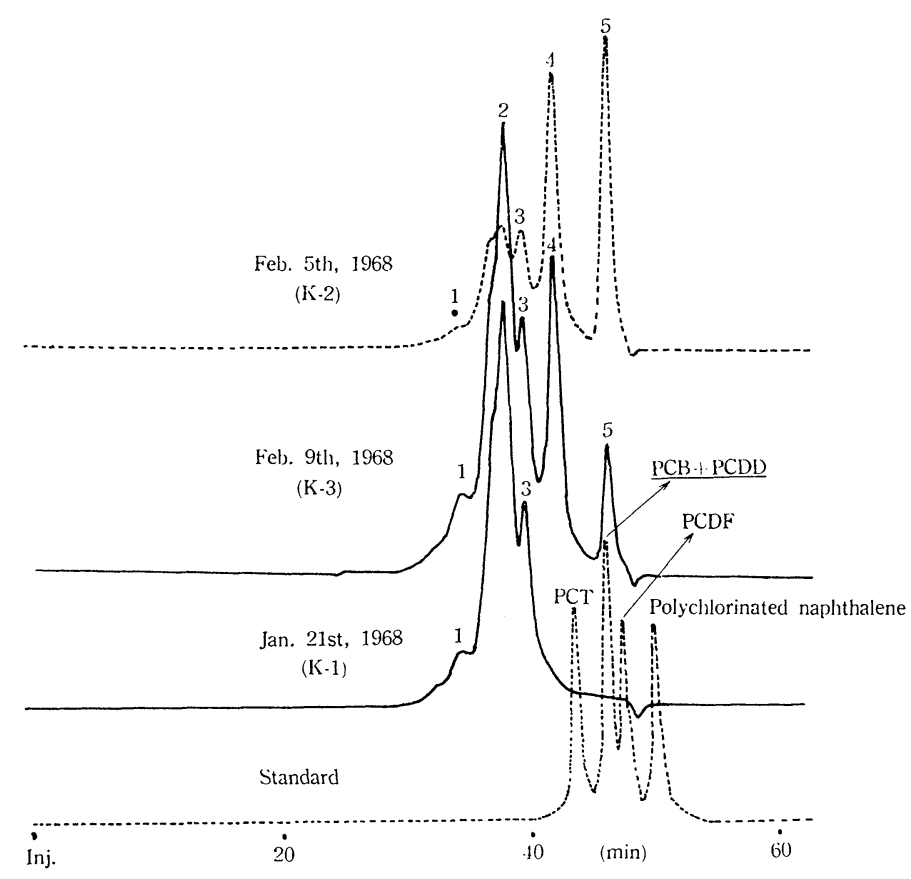

Fig. 1. Gel permeation chromatograms of the hexane extraction from saponified Kanemi rice oils

Column, Shimadzu HSG- $15 \times 2$; eluent, methylene chloride; flow rate, $0.6 \mathrm{ml} / \mathrm{min}$; detector, UV-254 nm

concentration of total chlorine of the same oils. The most of the chlorine in the original oils was recognized in the hexane extract obtained from saponified oil (Table 1). The hexane extract was a yellowish oily substance in which large quantities of hydrocarbons and chlorinated compounds were found by MS analysis.

Figure 1 showed gel permeation chromatograms of standards and the hexane extract from saponified samples. This gel permeation chromatography gave adequate resolution of standards, except one overlapping (PCB + PCDD). The oil which was manufactured on Jan. 21st in 1968 was not contaminated with PCB.

The chromatogram of the hexane extraction from this oil showed three peaks in which no chlorinated compounds were detected by MS analysis. The chromatograms of the extracts from the oils with PCB contamination showed five peaks. Compounds of peak 1,2 and 3 were similar to those in the chromatogram of non contaminated oil. The elution time of peak 4 was earlier than that of PCT, and MS analysis revealed that this peak was composed of hydro- carbons and high molecular organochlorinated compounds. The elution time of peak 5 corresponded to that of $\mathrm{PCB}$, and it was confirmed that this peak was composed of only PCB by MS analysis. The chromatogram of the oil- $(\mathrm{K}-4)$ was similar to that of the oil-(K-3). As shown in Fig. 1, the area ratios of peak 4 vs. peak 5 of the oil- $(\mathrm{K}-3)$ and $(\mathrm{K}-4)$ were much higher than that of the oil-(K-2). The eluates corresponding to peak 4 of each sample were collected, concentrated and cleaned up by thin layer chromatography. Figure 2 showed thin layer chromatograms of squalene, PCB, PCT and the eluates of three oils. These three eluates showed similar chromatograms each other, i.e., the lower spot was detected by both UV light and iodine vapour. MS analysis confirmed that the spot contained high molecular chlorinated compounds. The upper spot was only detected with iodine vapour, and the spot didn't contain any chlorinated compounds. The UV positive band of each sample was scraped off, eluted and analyzed by ECD-GC. As shown in Fig. 3, GC-analysis revealed that each sample was 


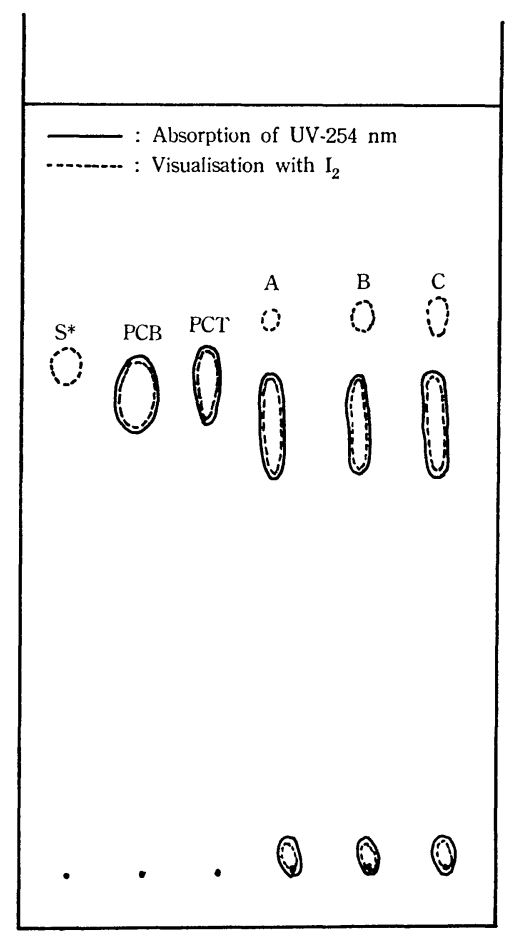

Fig. 2. Thin layer chromatograms of standards and the fourth eluates from gel permeation chromatography

TLC plate, Merck Kieselgel $60 \mathrm{~F}_{254}$; mobile phase, $n$-hexane

$\mathrm{A}, \mathrm{B}$ and $\mathrm{C}$ were the fourth eluated of the oils which were manufactured on Feb. 5,9 and 10,1968 , respectively.

$\mathrm{S}^{*}$, squalene

composed of many compounds whose R.T. were later than that of PCT (Aroclor 5460). The chromatograms of three samples were similar to each other.

The compounds corresponding to each peak were identified by GC-MS as shown in Table 2 . These compounds were composed of tetra- to deca-chlorinated quaterphenyl, hepta- to nonachlorinated quaterphenyl ether and penta- to octa-chlorinated terphenyl. GC-MS analysis also revealed that polychlorinated quaterphenyls (PCQs) occupied quantitatively the most of the unknown organochlorinated compounds, and the most of PCQs was occupied with hepta- and octa-chlorinated quaterphenyls. The mass spectra of peak 7 and 20 in the gas chromatogram were given in Fig. 4. Peak 7 was identified as hexa-, hepta- and octa-chlorinated quaterphenyls. Frag- ments of hexa-chlorinated quaterphenyl were as follows: The molecular ion $\left(\mathrm{M}^{+}\right)$was found at $m / e 544$, loss of double $\mathrm{Cl}$ yielded the peak at $m / e 474$, subsequently elimination of double $\mathrm{Cl}$ resulted in $m / e$ 404. The hexa- and octachlorinated quaterphenyls were identified in an analogous fashion as described above. The spectrum of peak 20 showed to contain heptato deca-chlorinated quaterphenyls and hepta- to nona-chlorinated quaterphenyl ethers. These chlorinated quaterphenyl ether (PCQE) fragments also showed an analogous fashion as PCQ fragments. Neutron activation analysis revealed that the chlorine concentrations of the unknown organochlorinated compounds in the oils- $(\mathrm{K}-2$, $\mathrm{K}-3$ and $\mathrm{K}-4)$ were 537, 287 and $343 \mathrm{ppm}$, respectively (Table 3 ). It was also clear that the most chlorine of the hexane extracts from saponified oils was derived from the unknown compounds and PCB. The result of analysis showed a surprising fact that the unknown organochlorinated compounds were contained 1.1 to 4.1 times more than PCB amount in Kanemi rice oils.

\section{Discussion}

From Feb. 5th to Mar. 22nd in 1968, the thermotransfer medium (KC-400) leaked out into Kanemi rice oil in the deodorization tank. The tank was maintained at about $230^{\circ} \mathrm{C}$ and $4 \mathrm{mmHg}$ pressure, and a high pressure steam was jetted into the tank in order to deodorize the oil. The component ratios of $\mathrm{PCB}$ in the oils were very different from that of original $\mathrm{KC}-400 .{ }^{4}$ ) The changes of $\mathrm{PCB}$ component and the ratios of PCDF vs. PCB concentration varied with the production date of the oil, i.e., in the oils produced on Feb. 9 and 10th in 1968, the component ratio of $\mathrm{PCB}$ was the most different from $\mathrm{KC}-400$ and the ratio of PCDF vs. PCB concentration became the highest. ${ }^{4)}$ The ratio of the unknown organochlorinated compounds vs. PCB concentration also showed a similar tendency as described above. Our earlier study made clear that PCDF was formed from PCB by heating at high temperature. ${ }^{7,8}$ The reason to believe those described above is that the thermotransfer medium might be heated at extraordinary high temperature or the deodorization of the oil might be carried out the vigorous condition for the two days. When tetra-chloro- 


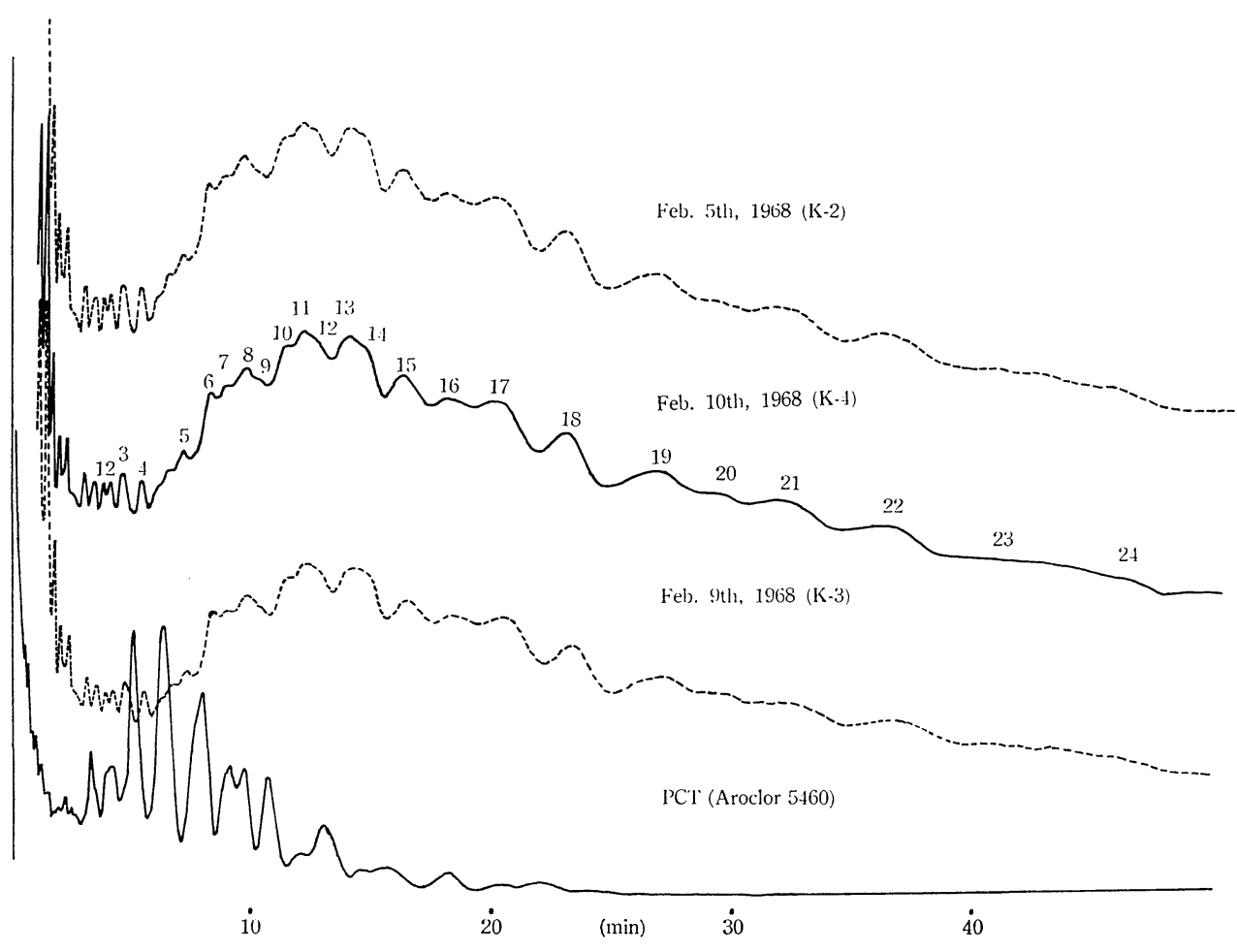

Fig. 3. Gas chromatograms of PCT (Aroclor 5460) and unknown organochlorinated compounds in Kanemi rice oils

GC, Varian Aerograph 2100; column, $2 \mathrm{~mm} \times 2 \mathrm{~m}$ glass column packed with $2 \%$ OV-210 on Gaschrom Q (100/120 mesh); injection, column and detection temp., 215 , 245 and $293^{\circ} \mathrm{C}$, respectively; detector, ${ }^{63} \mathrm{Ni}-\mathrm{ECD}$; carrier gas, $\mathrm{N}_{2}(30 \mathrm{ml} / \mathrm{min})$

Table 3. The Chlorine Concentration of Unknown Organochlorinated Compounds in Kanemi Rice Oils

\begin{tabular}{|c|c|c|c|c|c|c|}
\hline \multirow{2}{*}{ Production date } & \multicolumn{4}{|c|}{ Concentration of chlorine (ppm) } & \multirow{2}{*}{$\begin{array}{c}\text { Ratio } \\
\text { (UOC/PCB) }\end{array}$} & \multirow{2}{*}{$\begin{array}{c}\text { Ratio }^{7)} \\
(\mathrm{PCDF} / \mathrm{PCB})\end{array}$} \\
\hline & $\begin{array}{l}\text { Original } \\
\text { sample }\end{array}$ & $\begin{array}{l}\text { Hexane } \\
\text { extract }^{\text {a) }}\end{array}$ & $\mathrm{UOC}^{\mathrm{b})}$ & $\mathrm{PCB}^{\mathrm{c})}$ & & \\
\hline Feb. 5th, 1968 & 1,485 & 997 & 537 & 500 & 1.1 & 0.007 \\
\hline Feb. 9th, 1968 & 554 & 448 & 287 & 80 & 3.6 & 0.013 \\
\hline Feb. 10th, 1968 & 446 & 462 & 343 & 83 & 4.1 & 0.014 \\
\hline Jan. 21st, 1968 & 11 & 13 & & & & \\
\hline
\end{tabular}

a) Hexane extract from the saponified sample

b) Unknown organochlorinated compounds

c) The figures were calculated from the concentration of PCB with the method of the authors ${ }^{8)}$ 

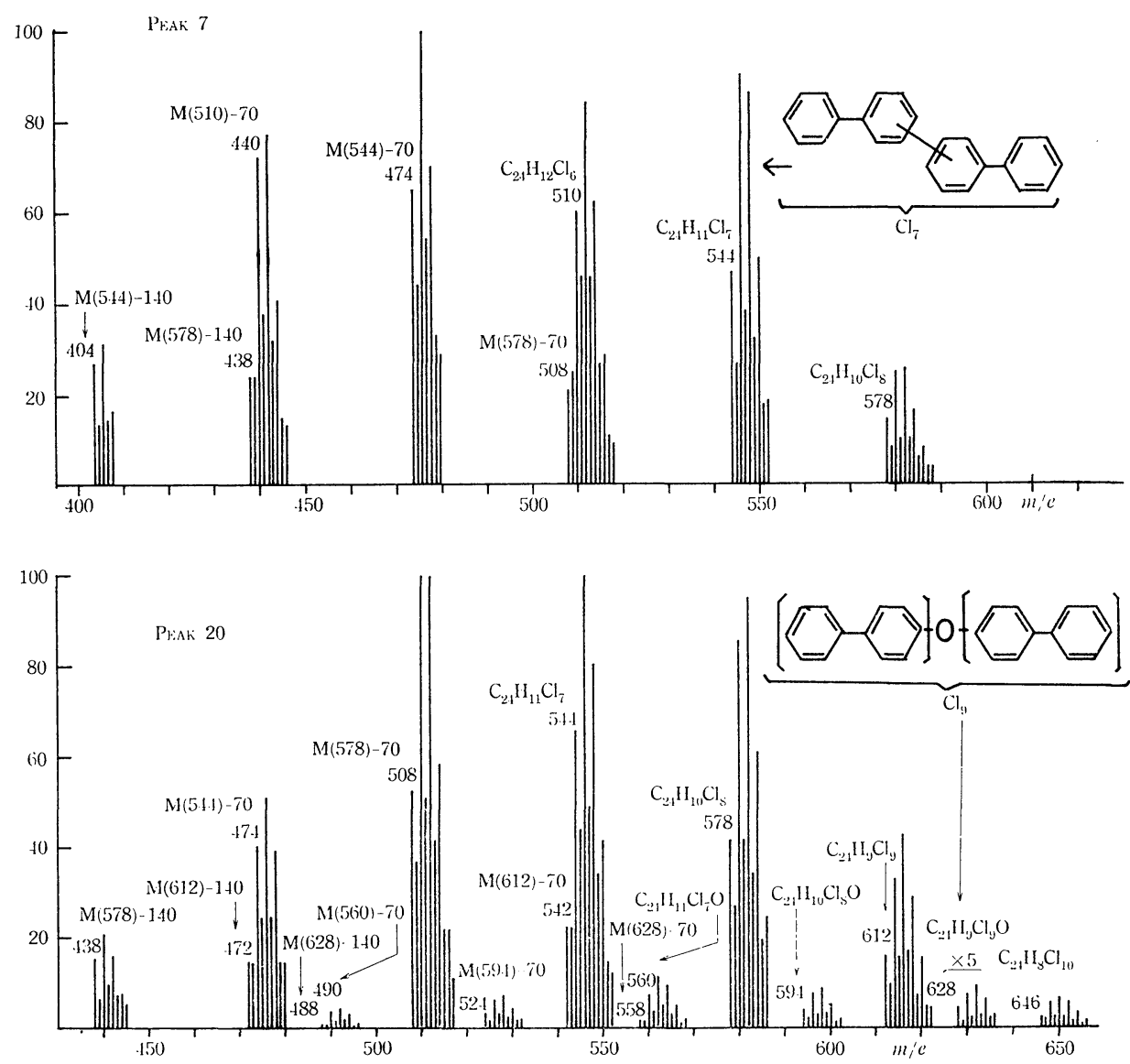

Fig. 4. Mass spectra of peak 7 and 20 in Fig. 3

GC-MS, Shimadzu LKB-9000;

GC-MS condition: ionizing energy, $20 \mathrm{eV}$; accel volt., $3.5 \mathrm{kV}$; chamber temp., $270^{\circ} \mathrm{C}$; column, $3 \mathrm{~mm} \times 1 \mathrm{~m}$ glass column packed with $2 \% \mathrm{OV}-210$ on Gaschrom $\mathrm{Q}$ $(100 / 120 \mathrm{mesh})$; in jection temp., $250^{\circ} \mathrm{C}$; column temp., $220^{\circ} \mathrm{C}$; carrier gass, $\mathrm{H}(30 \mathrm{ml} / \mathrm{min})$

biphenyl was irradiated in thin films with UV lamps or sunlight, hexa-, hepta- and octachlorinated quaterphenyls formed from original PCB. Moreover, undeca- and dodeca-chlorinated quaterphenyl generated on irradiating hexachlorobiphenyl. ${ }^{\text {) }}$ These PCQs formed presumably because of the proximity of chlorobiphenyl molecules to the photochemically generated aryl radical. ${ }^{\text {) }}$ PCQs in Kanemi rice oils also generated presumably by similar reaction.

This study revealed that the higher molecular chlorinated compounds, which would play an important role in the cause of the "Yusho," were present in Kanemi rice oils. Therefore, it became clear that the "Yusho" was different from sole PCB intoxication. Hereafter, it is necessary to conduct some biological and clinical studies condidering this important fact.

\section{Acknowledgement}

We thank for Mr. Akio Miyazaki, Osaka Agricultural Research Center, and Dr. Kenzi Tomura, the Institute for Atomic Energy, Rikkyo University, for assistance with GC-MS and neutron activation analysis, and Dr. Mitsuru Uchiyama, National Institute of Hygenic Science, and Dr. Masahiko Ueta, Kochi Prefectural Institute of Public Health, for supply of "Kanemi rice oils."

\section{References}

1) Tukamoto, H. et al.: Fukuoka Acta Med., 60, 496 (1969). 
2) Special report on the prevention, diagnosis and treatment for the "Yusho," Science and Technology Agency, p. 19 (1970).

3) Nagayama, J., Masuda, Y., Kuratsune, M.: Fukuoka Acta Med., 66, 593 (1975).

4) Miyata, H., Kashimoto, T., Kunita, N.: J. Food Hyg. Soc. Japan, 18, 260 (1977).

5) Yoshimura, T.: Fukuoka Acta Med., 62, 104 (1971).

6) Ugawa, M., Nakamura, A., Kashimoto, T.:
J. Food Hyg. Soc. Japan, 14, 415 (1973).

7) Miyata, H., Kashimoto, T.: Osaka Prefectural Institute of Public Health, ED. of Food Sanitation, 7, 65 (1976).

8) Miyata, H., Kashimoto, T.: J. Food Hyg. Soc. Japan, 19, 78 (1978).

9) Hutzinger, O., Jamieson, W. D., Safe, S., MacNeil, D., Zitko, V.: "The Chemistry of PCBs,” p. 121 (1974), CRC Press, Ohio. 